\title{
ANALYSIS OF INNER EAR POTASSIUM RECYCLING GENES AS POTENTIAL FACTORS ASSOCIATED WITH TINNITUS
}

\author{
MAŁGORZATA PAWEŁCZYK ${ }^{1}$, ELŻBIETA RAJKOWSKA루, PIOTR KOTYŁO, ADAM DUDAREWICZ ${ }^{1}$, \\ GUY VAN CAMP², and MARIOLA ŚLIWIŃSKA-KOWALSKA ${ }^{1}$ \\ ${ }^{1}$ Nofer Institute of Occupational Medicine, Łódź, Poland \\ Department of Audiology and Phoniatrics \\ ${ }^{2}$ University of Antwerp, Antwerp, Belgium \\ Department of Medical Genetics
}

\begin{abstract}
Tinnitus is defined as a perception of sound in the absence of an external acoustic stimulus. Several factors are known to influence tinnitus, e.g. hearing loss, noise exposure, age, and hypertension. As only certain individuals develop tinnitus in the presence of the above risks and in approximately $50 \%$ of cases tinnitus is not attributed to any particular cause, the question arose whether this inter-individual susceptibility to tinnitus could be explained by the influence of genetic factors. Objectives: To test the hypothesis that genetic variability in genes of the potassium recycling pathway is associated with increased susceptibility to tinnitus. Materials and Methods: The study group consisted of 626 subjects exposed to occupational noise (128 with tinnitus and 498 without tinnitus). 99 single nucleotide polymorphisms were investigated in 10 genes involved in the potassium recycling pathway in the inner ear, previously selected as putative noise-induced hearing loss (NIHL) candidate genes. Results: Nominally significant associations were obtained for 2 variants in KCNE1 (potassium voltage-gated channel, Isk-related family, member 1) and SLC12A2 (solute carrier family 12, member 2) genes. The first gene contributed to tinnitus that developed independently of hearing loss, while the second one was associated with increased susceptibility to noise-induced hearing loss. Conclusions: Present findings lend support to the notion of potassium recycling pathway genes as possible risk modifiers of tinnitus in individuals with and without hearing loss. Due to the lack of replication in other independent populations these results should be seen as suggestive.
\end{abstract}

Key words:

KCNE1, Noise-induced hearing loss, Potassium recycling pathway genes, SLC12A2, Tinnitus

\section{INTRODUCTION}

Tinnitus is defined as a perception of sound in the absence of an external acoustic stimulus. Estimates suggest that approximately $10-14 \%$ of people in the general population and up to $30 \%$ of the subjects in a population aged 55 years and older report the presence of tinnitus $[1,2]$.
Up to now the mechanisms leading to the occurrence of tinnitus remain unclear. Several theories have been proposed, but the lack of consensus reflects the complex nature of this condition. Tinnitus possibly originates as a result of hair cell damage which leads to an aberrant stimulation of the cochlear nerve. This, in turn, may be processed by the auditory cortex and perceived as a specific

The study was supported by the Ministry of Science and Higher Education (grant IMP/18.3/2009), Poland.

Received: June 29, 2012. Accepted: October 25, 2012.

Address reprint request to M. Pawełczyk, Department of Audiology and Phoniatrics, Nofer Institute of Occupational Medicine, św. Teresy 8, 91-348 Łódź, Poland (e-mail:mmalczyk@imp.lodz.pl). 
sound with a characteristic frequency [3]. Another theory states that tinnitus is a result of changes in the central nervous system, where dynamic, multiple, parallel, and overlapping brain networks are at stake, which include sensory auditory areas together with cortical regions involved in perceptual, emotional, mnemonic, attentional, and salience functions [4].

Several factors are known to influence tinnitus. The best documented are: hearing loss and noise exposure, but additionally age, ototoxic drugs, head injury, dietary factors, hypertension, elevated blood lipids and alcohol consumption should be mentioned as well [5]. However only certain individuals develop tinnitus in the presence of the above risks and in approximately 50\% of cases tinnitus is not attributed to any particular cause [6]. Moreover, in some individuals tinnitus develops progressively, while in others it has a sudden onset. This individual susceptibility to tinnitus may be explained by the influence of different environmental and intrinsic factors, including genetic ones.

Identification of genetic factors underlying tinnitus encounters many problems like phenotypic heterogeneity, variable penetrance, contradictory data on heritability and very limited information concerning the early onset of this condition. Moreover, the first genes that are regarded as potentially causative ones are related mainly to monogenic disorders associated with tinnitus as a secondary symptom rather than with a chronic primary tinnitus. These are $\mathrm{COCH}$ in autosomal dominant nonsyndromic sensorineural deafness, GLA in Fabry disease or NF2 in neurofibromatosis type II [7].

Different strategies are being chosen in genetic investigations in order to discover markers leading to a complex disease such as tinnitus. The commonly used one is a 'candidate gene approach' which is based on the prior knowledge of the physiology and biology underlying the disorder being studied. Although the exact mechanisms of tinnitus have not been revealed yet, its frequent association with hearing loss can be the basis for the selection of candidate genes. However, although a number of observations favored a prominent role of the serotonergic system in the auditory perception and in the etiology of tinnitus [8], negative associations of tinnitus with serotonin receptor genes $1 \mathrm{~A}$ and $3 \mathrm{~A}[9,10]$ and serotonin transporter gene have been documented [11]. The only detected positive associations concern two genes that encode neurotrophic factors. The risk of developing tinnitus in conjunction with hearing impairment is significantly reduced in carriers of the Met in Val66Met variant of the gene encoding brainderived neurotrophic factor $(B D N F)$ [12]. Combining the data for $B D N F$ gene with the results obtained in the gene encoding glial cell-derived neurotrophic factor $(G D N F)$ revealed that $16 \%$ of the variance in tinnitus severity could be explained [13].

As tinnitus prevalence is considerably higher in noiseexposed populations and a significant correlation between tinnitus and occupational noise has been found [1], one may propose a hypothesis that variability in genes involved in the development of noise-induced hearing loss (NIHL) may play a key role in determination of individual susceptibility also in case of tinnitus. Previously we have performed a number of associations studies that aimed at identification of NIHL susceptibility genes. Among carefully selected candidate genes, those regulating potassium recycling in the inner ear seemed to be of great importance, as evidenced by the fact that mutations in $\mathrm{K}+$ channel genes in the inner ear often lead to hearing loss in animals and humans [14-18]. Two genes (KCNE1 - potassium voltage-gated channel, Isk-related family, member 1 and $K C N Q 4$ - potassium voltage-gated channel, KQT-like subfamily, member 4) were finally reported to be associated with NIHL in Polish and Swedish populations [19,20].

To test the hypothesis that genetic variability in genes of the potassium recycling pathway is associated with increased susceptibility to tinnitus, we have performed 
a pilot study analyzing Single Nucleotide Polymorphisms (SNPs) in selected GJB (gap junction protein beta) genes and $\mathrm{K}+$ channel/transporter genes in a large sample set of Polish individuals exposed to occupational noise.

\section{MATERIALS AND METHODS}

\section{Subjects}

The study group consisted of the noise-exposed male subjects selected for the purpose of previous association analysis aiming at identification of NIHL susceptibility genes. A detailed description of the methods used to select subjects has been published previously $[20,21]$. Briefly, the study group was drawn from a database of over 3860 entries from the noise-exposed workers. Information on these subjects was gathered by the use of a questionnaire, which requested information on general health state, hereditary factors and noise exposures. All subjects with a history of hearing loss in family, extended treatment with aminoglycosides, middle ear diseases, conductive hearing loss, meningitis, acoustic trauma and the difference between the right and the left ear exceeding $40 \mathrm{~dB}$ were excluded from the study. For each subject a Z-score that corrects for age, gender, tenure of exposure and noise exposure level, was generated based on the ISO 1999 (ISO 1999 - International Organization of Standardization, 1990). Subsequently, based on the mean hearing thresholds (HTL) for the left ear at 4 and $6 \mathrm{kHz}$, which are the frequencies most sensitive to noise trauma [22], we selected $20 \%$ most resistant (with better HTLs than predicted in the ISO model) and $20 \%$ most sensitive (with worse HTLs than predicted in the ISO model) subjects. All individuals answered the (Yes/No) question "Do you suffer from tinnitus?". This resulted in selection of 626 subjects - 128 with tinnitus (mean age: 42 years) and 498 without tinnitus (mean age: 41 years). The number of subjects with and without tinnitus in selected subgroups (resistant and sensitive to noise) is presented in Table 1.

The study protocol was approved by the Regional Bioethical Committee at the Nofer Institute of Occupational Medicine in Łódź, Poland.

\section{SNP selection and genotyping}

Ten potassium recycling pathway genes were selected as candidate tinnitus susceptibility genes, as it was described for NIHL candidate genes [19,20]. Briefly, SNPs were initially selected from the complete list of SNPs available in the NCBI database (URL: http://www.ncbi. nlm.nih.gov) along with the entire length of the gene. Each gene was covered with at least two SNPs with an inter SNP distance of approximately $10 \mathrm{~Kb}$. Coding SNPs were preferred over intronic ones and priority was given to SNPs with heterozygosity values of at least 0.4 . In the second phase of the study the list of SNPs was extended according to the information provided by the HAPMAP database (http://www.hapmap.org). This data was subsequently used by MARKER (http://www.gmap.net/marker) to select tagSNPs. The new list of SNPs was checked by Illumina (Illumina, San Diego, CA, USA, www.Illumina.com) in respect of their appropriate designability

Table 1. Subjects qualified for genetic analysis

\begin{tabular}{lccc}
\hline \multicolumn{1}{c}{ Disease state } & $\begin{array}{c}\text { Whole database } \\
\mathrm{n}(\%)\end{array}$ & $\begin{array}{c}\text { Susceptible to noise subjects } \\
\mathrm{n}(\%)\end{array}$ & $\begin{array}{c}\text { Resistant to noise subjects } \\
\mathrm{n}(\%)\end{array}$ \\
\hline With tinnitus & $128(20.4)$ & $89(28.4)$ & $39(87.5)$ \\
Without tinnitus & $498(79.6)$ & $224(71.6)$ & $274(12.5)$ \\
Total & $626(100.0)$ & $313(100.0)$ & $313(100.0)$ \\
\hline
\end{tabular}


score. SNPs which did not meet these expectations were replaced by other informative SNPs indicated after the Tagger analysis [23].

In total 99 SNPs were genotyped in 10 potassium recycling genes. Among them 33 SNPs were genotyped with AcycloPrime Fluorescence Polarization (FP) SNP Detection System (Perkin Elmer Life Sciences, Boston, MA, USA) as described before [19]. SNPs, for which no assay could be developed with the FP methods, were genotyped with the SNaPshot detection system (Applied Biosystems, Foster City, CA, USA), as described previously [24]. Additionally 66 SNPs were genotyped by Illumina. An overview of all SNPs selected for this study was described in our previous study [20].

\section{Data quality control}

Prior to the selection of 626 subjects for the purpose of genetic analysis, several data quality control steps were performed in order to eliminate samples not meeting quality standards. All subjects with more than $10 \%$ missing genotypes and all SNPs with a failure rate above $4 \%$ were excluded from the study. Additionally all individuals with an outlying genetic background when compared to the rest of population were removed after running the CHECKHET programme [25]. Unknown family relationships and sample duplications were eliminated after running the GRR program [26]. All SNPs were calculated for HardyWeinberg equilibrium using Genotype Transposer [27].

\section{Statistical analysis}

Hearing threshold levels were compared between subjects with and without tinnitus with non-parametric MannWhitney $U$ test. The effect of genotype on tinnitus was tested within a logistic regression framework with a correction for noise exposure and age as confounding factors. We assumed an additive genetic model where alleles were treated as continuous covariate. All calculations were performed in two steps. In the first step the effect of genotype on the disease status was checked in all subjects - susceptible and resistant to noise. In the second step the effect of genotype was calculated for all significant results from the first step and it was performed separately in the susceptible to noise individuals (i.e. having hearing loss) and in the resistant to noise subjects (i.e. without hearing loss). P-values at the level of 5\% were regarded as significant. All calculations were performed with SPSS 12.0 software (SPSS Inc., Chicago, IL, USA; http://www.spss.com).

\section{RESULTS}

The audiometric data indicated that in the resistant to noise subgroup mean $( \pm 1$ STD) HTs were of normal values (better than $20 \mathrm{~dB} \mathrm{HL}$ ) and that HTs did not differ between subjects with and without tinnitus (Figure 1). In the second - susceptible to noise subgroup, HTs were worse than predicted by the ISO 1999:1990 model (Figure 1). Additionally, individuals with tinnitus had significantly worse HTs in all frequencies except $6 \mathrm{kHz}$ than the subjects without tinnitus (Mann Whitney U test).

Genetic analysis comprised 99 SNPs in 5 connexin genes (Cx26 (GJB2), Cx30 (GJB6), Cx30.3 (GJB4), Cx31 (GJB3), Cx32 (GJB1)), 4 potassium channels or channel subunits (KCNJ10 - potassium inwardly-rectifying channel, subfamily J, member 10, KCNQ4, KCNE1, KCNQ1 - potassium voltage-gated channel, KQT-like subfamily, member 1, and one $\mathrm{Na}^{+} / 2 \mathrm{Cl}^{-} / \mathrm{K}^{+}$cotransporter (SLC12A2 - solute carrier family 12 , member 2 ) which are involved in potassium recycling in the inner ear. Significant results obtained in statistical analysis are presented in Table 2.

The first step of the statistical analysis resulted in significant P-values for $1 \mathrm{SNP}$ (rs915539) in KCNE1 ( $\mathrm{p}=0.018$ ) and 1 SNP (rs10089) in SLC12A2 gene ( $\mathrm{p}=0.026)$. The subsequent analysis revealed significant associations for $K C N E 1$ in the resistant to noise subjects ( $\mathrm{p}=0.005$ ) and for $S L C 12 A 2$ gene in the susceptible to noise subjects $(\mathrm{p}=0.016)$. 
Table 2. Statistical results for single SNP analysis - the comparison of genotype frequencies between subjects with tinnitus and without tinnitus in the whole study group, in susceptible to noise group and in resistant to noise group

\begin{tabular}{ccccccccc}
\hline \multirow{2}{*}{ Gene } & \multirow{2}{*}{ SNP } & \multirow{2}{*}{ Genotype } & \multicolumn{2}{c}{ All cases } & \multicolumn{2}{c}{ Resistant cases } & \multicolumn{2}{c}{ Susceptible cases } \\
\cline { 4 - 9 } & & & $\mathrm{p}$ & OR (95\% CI) & $\mathrm{p}$ & OR $(95 \% \mathrm{CI})$ & $\mathrm{p}$ & OR (95\% CI) \\
\hline KCNE1 & rs915539 & AA & $\mathbf{0 . 0 1 8}$ & $\mathbf{1 . 3 4 4 2}$ & $\mathbf{0 . 0 0 5}$ & $\mathbf{1 . 8 8 1 9}$ & 0.403 & 1.1230 \\
& & AC & & $(\mathbf{1 . 0 2 4 2 - 1 . 7 6 4 2 )}$ & & $(\mathbf{1 . 1 9 5 8 - 2 . 9 6 1 6 )}$ & & $(0.7890-1.5982)$ \\
& & CC & & & & & & \\
\multirow{3}{*}{ Slc12A2 } & rs10089 & CC & $\mathbf{0 . 0 2 6}$ & 1.6293 & 0.669 & 1.2639 & $\mathbf{0 . 0 1 6}$ & $\mathbf{1 . 6 8 9 9}$ \\
& & CT & & $(1.0297-2.5532)$ & & $(0.4504-3.4604)$ & & $\mathbf{( 0 . 9 9 9 1 - 2 . 8 5 8 2 )}$ \\
& & & & & & & \\
& & & & & & & & \\
\hline
\end{tabular}

OR - odds ratio, CI - confidence interval.

P-values at the level of 5\% were regarded as significant (indicated in bold).

All calculations were adjusted for age and noise exposure level.
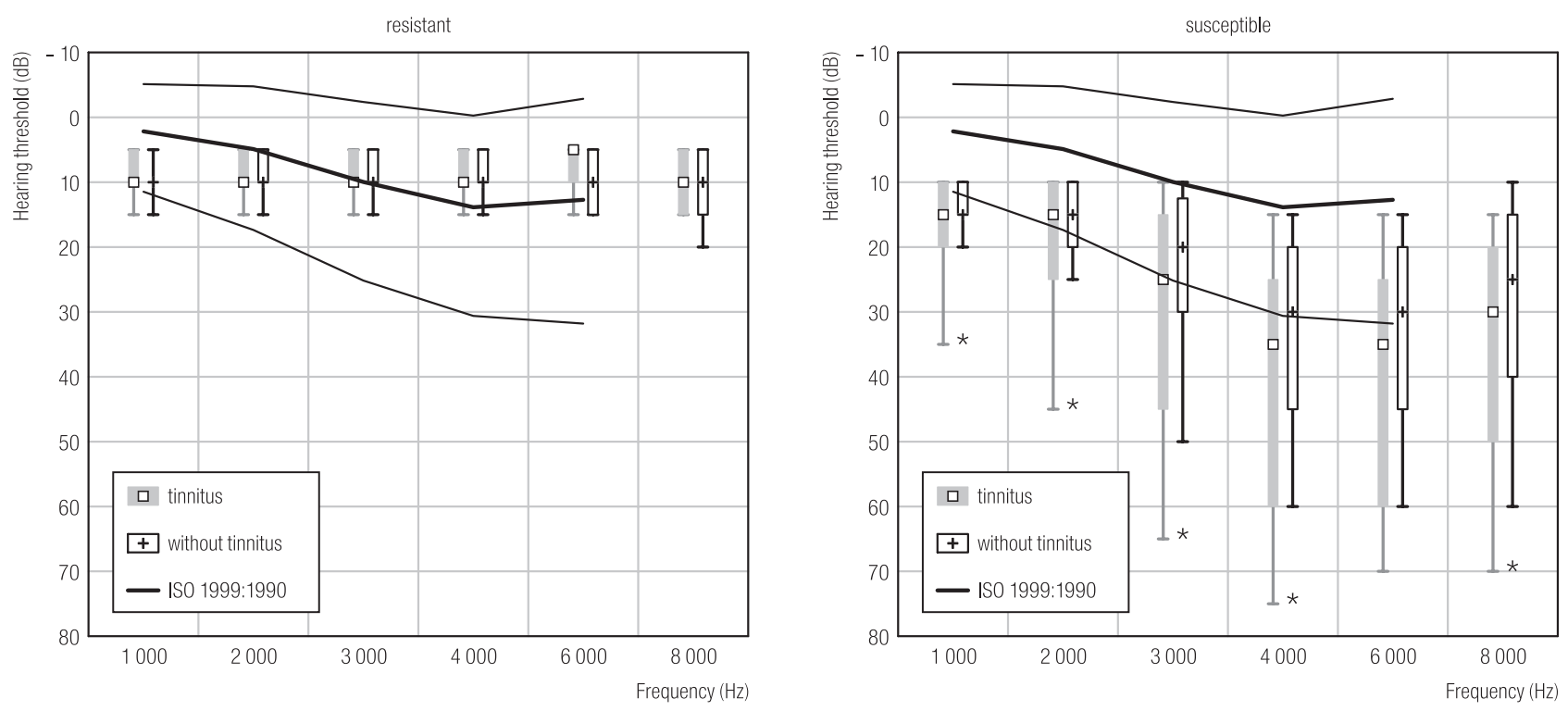

Significant difference $(\mathrm{p} \leq 0.05)$ between mean HTs in the subjects with and without tinnitus has been marked with asterisk (Mann-Whitney U test).

Fig. 1. Audiograms of the subjects with and without tinnitus, classified to susceptible or resistant to noise group against ISO 1999:1990 model predictions

\section{DISCUSSION}

Although the search for genetic factors predisposing to the development of tinnitus have recently become a focus of public awareness, the inter-individual differences in severity and course of the symptoms have not been explained yet. The involvement of tinnitus innate factors has been analyzed in several familial aggregation studies.

Hendrickx et al. [5] in a multicentre study involving 198 families from 7 European countries reported that subjects related to a sibling with tinnitus were 1.7 times more likely to have tinnitus themselves than subjects from a family without tinnitus. By contrast, Kvestad et al. [28], using 
a large population-based cohort of Norwegian nuclear families, reported a heritability of 0.11 , suggesting that additive genetic effects explain only a small proportion of the variance of tinnitus in the population. However the lack of attention to wording while specifying the tinnitus-related complaints (i.e. the questionnaire including the questions about bothersome tinnitus with response categories: yes, no, and don't know/maybe), has been suggested as a weakness of this study [29].

Identification of familial aggregation of tinnitus opened the door to specific studies aiming at identification of genetic factors. In our previous study aimed at identification of susceptibility genes for noise-induced hearing loss we found that SNPs in GJB1, GJB2, GJB4, KCNJ10, KCNQ1, KCNE1 and KCNQ4 genes may play an important role in Polish population. Two of them, namely KCNE1 and KCNQ4 were previously reported also in a Swedish sample set. Considerably higher prevalence of tinnitus in noise-exposed populations and significant correlation between tinnitus and occupational noise suggested that previously identified noise-induced hearing loss (NIHL) susceptibility genes may also be involved in the pathophysiology of tinnitus. Since tinnitus may be caused by hair cell damage which leads to an aberrant stimulation of the cochlear nerve, genes responsible for potassium recycling in the inner ear, i.e. providing the major charge carrier for the sensory transduction, constitute a particularly interesting group for an association analysis.

Taking advantage of our previous studies on susceptibility factors contributing to NIHL, in this study we were able to apply a unique approach to identify genetic polymorphisms that possibly contribute to tinnitus separately in the subjects with and without hearing loss. As it is known that among all tinnitus patients only a small proportion (of about 10\%) do not exhibit hearing loss in regular audiometric examination, the analysis of the resistant to noise group in this study ( 39 subjects) is therefore of great importance to identify genetic polymorphisms contributing to tinnitus which is not a consequence of hearing damage. In the resistant to NIHL group no differences in HTs were observed between the subjects with and without tinnitus, thereby confirming tinnitus independence of cochlear damage. On the other hand such a difference between the patients with and without tinnitus was observed in the susceptible to NIHL subgroup. These data suggest that the subjects most susceptible to NIHL, i.e. with the greatest HTs, are also the most sensitive to develop tinnitus.

The main aim of this study was to investigate whether genetic variability in the potassium recycling pathway genes underlies an increased susceptibility to the development of tinnitus in Polish population. One could argue that corrections for multiple testing should be applied to keep the type I error under control. However genetic epidemiologists have not yet come to a consensus how to do it when investigating a small subset of genes. The Bonferroni correction uses the number of independent hypotheses to correct the p-value. In this study we have analyzed multiple SNPs per gene, many of them being in linkage disequilibrium (LD). These tests cannot be therefore considered as independent ones and for this reason Bonferroni correction has not been applied here, as being in our opinion too strict. Instead we propose to treat all the significant results from this study as nominally significant ones and as suggestions for further analysis with higher power to detect a causative variant.

Nominally significant associations for the whole study group indicated 2 genes as possible tinnitus innate factors: KCNE1 and SLC12A2. For both genes nominally significant associations have been replicated in the analyzed subgroups, in the susceptible to noise group for SLC12A2 and in the resistant to noise group in KCNE1 respectively. One may assume that significant result for the rs10089 variant in SLC12A2 gene in the subjects with hearing 
impairment may be associated with hearing loss and not tinnitus itself. However, previous analysis did not report SLC12A2 as NIHL susceptibility gene neither in our Polish [20] nor in the Swedish population [19]. Given the special importance of the subjects with tinnitus and normal hearing, in our opinion the results obtained for KCNE1 are particularly interesting. Previously KCNE1 has been proposed as NIHL susceptibility gene in Polish and Swedish populations [20]. Although tinnitus often coexists with noise-induced hearing loss, according to the results of this study, the associated genetic variants are not overlapping - in case of tinnitus it is rs915539 and in case of NIHL it is rs2070358. The major weakness of this study is the lack of specification of tinnitus-related complaints. The results are based solely on a yes/no answer to a single question about the presence of tinnitus, and we are aware of the limitations that result from this fact. A specific study with a detailed phenotypic analysis of tinnitus would obviously be preferred, but we feel that a pilot study as the current one can also be very valuable.

Nowadays association signals are only considered valid when they are replicated in an independent sample set. Although replication at the gene level is regarded as sufficient [30], replication at the SNP level is considered to be stronger. Recently Sand et al. [31] hypothesized that primary chronic tinnitus could be a part of the phenotypic spectrum associated with KCNE1. Systematic screening of coding variants in the open reading frame and in the adjacent sequence in subjects who had experienced tinnitus for a minimum six months did not, however, confirm this hypothesis. Since the vast majority ( $>80 \%$ ) of associated variants may fall outside coding regions, the importance of including both coding and non-coding regions in the search for disease associated genes has been emphasized [32]. Therefore, the lack of significant differences in KCNE1 coding variants of the control subjects and the tinnitus patients does not, in our opinion, disprove this gene as a risk factor in a complex pathology such as tinnitus.
To conclude, the present findings lend support to the notion of potassium recycling pathway genes as possible risk modifiers of tinnitus in individuals with and without hearing loss. However further genetic analysis in other independent and carefully selected populations is required. Identification of genetic factors that predispose to the development of tinnitus will hopefully open the door to possible new therapeutic approaches for tinnitus patients.

\section{REFERENCES}

1. Rosenhall U, Karlsson AK. Tinnitus in old age. Scand Audiol 1991;20:165-71.

2. Sindhusake D, Mitchell P, Newall P, Golding M, Rochtchina E, Rubin G. Prevalence and characteristics of tinnitus in older adults: the Blue Mountains Hearing Study. Int J Audiol 2003;42:289-94.

3. Ahmad N, Seidman M. Tinnitus in the older adult. Epidemiology, pathophysiology and treatment options. Drugs Aging 2004;21(5):297-305.

4. Elgoyhen AB, Langguth B, Vanneste S, De Ridder D. Tinnitus: network pathophysiology-network pharmacology. Front Syst Neurosci 2012;6:1-12.

5. Hendrickx J-J, Huyghe JR, Demeester K, Topsakal V, Van Eyken E, Fransen E, et al. Familial aggregation of tinnitus: A European multicentre study. B-ENT 2007;3(7):51-60.

6. Stouffer JL, Tyler RS. Characterization of tinnitus by tinnitus patients. J Speech Hear Disord 1990;55:439-53.

7. Sand PG, Langguth B, Kleinjung T, Eichhammer P. Genetics of chronic tinnitus Prog Brain Res 2007;166:159-68.

8. Simpson JJ, Davies WE. A review of evidence in support of a role for 5-HT in the perception of tinnitus. Hear Res 2000;145 (1-2):1-7.

9. Kleinjung T, Langguth B, Fischer B, Hajak G, Eichhammer P, Sand PG. Systematic screening of the Serotonin Receptor $1 A$ (5HT1A) gene in chronic tinnitus. J Otology 2006;2: $83-5$. 
10. Kleinjung T, Fischer B, Vielsmeier V, Langguth B, Sand P. Serotonin-Rezeptor 3A-Genvarianten bei Patienten mit chronischem Tinnitus. 79th Annual Meeting of the German Society of Oto-Rhino-Laryngology, 2008 Bonn.

11. Sand P, Langguth B, Kleinjung T, Fischer B, Stoertebecker P, Pfluegl S, et al. Investigation of the 5-HTTLPR in chronic tinnitus. Am J Med Genet 2006a;141B:763.

12. Sand P, Kleinjung T, Langguth B, Eichhammer P, Fischer B, Pratt $\mathrm{C}$, et al. Investigation of a BDNF missense variant in chronic tinnitus. Int J Neuropsychopharmacol 2006b;9:S1.

13. Kleinjung T, Frank E, Vielsmeier V, Landgrebe M, Langguth B, Sand P. BDNF and GDNF variants predict tinnitus severity Otolaryngol head Neck Surg 2009;141 Suppl 1:180-1.

14. Neyroud N, Tesson F, Denjoy I, Leibovici M, Donger C, Barhanin $\mathrm{J}$, et al. A novel mutation in the potassium channel gene KVLQT1 causes the Jervell and Lange-Nielsen cardioauditory syndrome. Nat Genet 1997;15:186-9.

15. Kubisch C, Schroeder BC, Friedrich T, Lutjohann B, ElAmraoui A, Marlin S, et al. KCNQ4, a novel potassium channel expressed in sensory outer hair cells, is mutated in dominant deafness. Cell 1999;96:437-46.

16. Flagella M, Clarke LL, Miller ML, Erway LC, Giannella RA, Andringa A, et al. Mice lacking the basolateral $\mathrm{Na}$ $\mathrm{K}-2 \mathrm{Cl}$ cotransporter have impaired epithelial chloride secretion and are profoundly deaf. J Biol Chem 1999;274:26946-55.

17. Marcus DC, Wu T, Wangemann P, Kofuji P. KCNJ10 (Kir4.1) potassium channel knockout abolishes endocochlear potential. Am j Physiol Cell Physiol 2002;282:C403-7.

18. Del Castillo J, Villamar M, Moreno-Pelayo MA, Del Castillo FJ, Alvarez A, Tellesia D, et al. A deletion involving the connexin 30 gene in nonsyndromic hearing impairment. N Engl J Med 2002;346(4):243-49.

19. Van Laer L, Carlsson PI, Ottschytsch N, Bondeson ML, Konings A, Vandevelde A, et al. The contribution of genes involved in potassium-recycling in the inner ear to noise-induced hearing loss. Hum Mutat 2006;27:786-95.

20. Pawelczyk M, Van Laer L, Fransen E, Rajkowska E, Konings A, Carlsson P-I, et al. Analysis of gene polymorphisms associated with $\mathrm{K}+$ ion circulation in the inner ear of patients susceptible and resistant to noise-induced hearing loss. Ann Hum Genet; 2009;73:411-21.

21. Śliwińska-Kowalska M, Dudarewicz A, Kotyło P, Zamysłowska-Szmytke E, Pawlaczyk-Łuszczyńska M, Gajda-Szadkowska A. Individual susceptibility to noise-induced hearing loss: choosing an optimal method of retrospective classification of workers into noise-susceptible and noise-resistant groups. Int J Occup Med Environ Health 2006;19:235-45. DOI: 10.2478/ v10001-006-0029-2.

22. Pirila T. Left-right asymmetry in the human response to experimental noise exposure. I. Interaural correlation of the temporary threshold shift at $4 \mathrm{kHz}$ frequency. Acta Otolaryngol 1991;111(4):677-83.

23. De Bakker PI, Yelensky R, Pe'er I, Gabriel SB, Daly M, Altshuler D. Efficiency and power in genetic association studies. Nat Genet 2005;37:1217-23.

24. Van Eyken E, Van Laer L, Fransen E, Topsakal V, Lemkens N, Laureys W, et al. KCNQ4: a gene for age-related hearing impairment? Hum Mutat 2006;27:1007-16.

25. Curtis D, North BV, Gurling HMD, Blaveri E, Sham PC. A quick and simple method for detecting subjects with abnormal genetic background in case-control samples. Ann Hum Genet 2002;66:235-44.

26. Abecasis GR, Cherny SS, Cookson WOC, Cardon LR. GRR: graphical representation of relationship errors. Bioinformatics 2001;17:742-3.

27. Cox DG, Canzian F. Genotype transposer: automated genotype manipulation for linkage disequilibrium analysis. Bioinformatics 2001;17:738-9.

28. Kvestad E, Czajkowski N, Engdahl B, Hoffman H, Tambs K. Low heritability of tinnitus. Arch Otolaryngol Head Neck Surg 2010;136(2):178-82.

29. Sand PG. Genetic risk factors in chronic tinnitus. In: Møller AR, Langguth, B. DeRidder D, Kleinjung T, editors. Textbook of tinnitus. New York: Springer Science + Business Media, LLC; 2011. p. 47-50. DOI 10.1007/978-160761-145-5_7. 
30. Neale BM, Sham PC. The future association studies: genebased analysis and replication. Am J Hum Genet 2004;75: $353-62$.

31. Sand PG, Luettich A, Kleinjung T, Hajak G, Langguth B. An examination of KCNE1 mutations and common variants in chronic tinnitus. Genes 2010;1:23-37.
32. Manolio TA, Collins F, Cox NJ, Goldstein DB, Hindorff LA, Hunter DJ, et al. Finding the missing heritability of complex diseases. Nature 2009;461(7265):747-53.

This work is available in Open Access model and licensed under a Creative Commons Attribution-NonCommercial 3.0 Poland License - http://creativecommons.org/ licenses/by-nc/3.0/pl/deed.en. 